\title{
Elementary Solutions of the Transport Equation and Their Applications*
}

\author{
K. M. CASE \\ Department of Physics, The University of Michigan, Ann Arbor, Michigan
}

\begin{abstract}
A new method of treating problems involving the transport equation is discussed. Starting from Van Kampen's observation that it is sufficient that "solutions" be distributions, the elementary solutions of the homogeneous equation are considered. These are found to have completeness and, in some cases, orthogonality properties which lead to the solution of more interesting problems by a conventional eigenfunction expansion. While the method is illustrated here with the simplest examples of neutron diffusion, it seems to be generally applicable.
\end{abstract}

\section{INTRODUCTION}

We wish to present here a new method for solving the transport equations describing neutron diffusion. The motivation is threefold. First, conventional methods of treatment, such as converting to integral equations, become extremely complicated for anything but a single uniform medium. Moreover, the solutions obtained for the integral equations are usually expressed as contour integrals. These are put in a tractable form for numerical transformation only after many transformations. It is desirable to find the transformed forms directly. Secondly, it may be hoped that an alternate approach will throw light on the general subject and suggest new methods of approximation. Lastly, the usual methods of obtaining rigorous solutions of particular problems are quite varied and seem to have no common bases. Especially the elementary approach familiar in the treatment of partial differential equations in which variables are separated and solutions expanded in normal modes seems lacking.

The last remark contains the essence of the method to be discussed. It is suggested by Van Kampen's work (1) on the related problems of plasma oscillations. Van Kampen makes two important points. First he notes that in problems involving the transport equation it is sufficient that admissible solutions be distributions in the sense of Schwartz. Secondly he shows that for the particular problem of a plasma these eigen-distributions are complete. Here it will be shown that similar (but more comprehensive) completeness properties hold for various

\footnotetext{
* Supported in part by the Office of Naval Research, U. S. Navy Department.
} 
neutron diffusion problems. In addition an orthogonality property is found which simplifies some expansions.

For illustration, attention here is restricted to the simplest problems of neutron diffusion. Thus only neutrons of one energy are considered. The density of neutrons at position $\mathbf{r}$ and velocity direction $\boldsymbol{\Omega}$ is then described by function $\psi(\mathbf{r}, \boldsymbol{\Omega}, t)$ which satisfies the equation

$$
\frac{\partial \psi}{\partial t}+v \mathbf{\Omega} \cdot \nabla \psi+v \sigma \psi=\frac{c}{4 \pi} v \sigma \int f\left(\boldsymbol{\Omega} \cdot \boldsymbol{\Omega}^{\prime}\right) \psi\left(\mathbf{r}, \mathbf{\Omega}^{\prime}, t\right) d \mathbf{r}^{\prime}+Q(\mathbf{r}, \mathbf{\Omega}, t) .
$$

$\sigma$ is the total cross section at velocity $v, c$ is the net number of neutrons produced per collision, and $Q$ describes the production of neutrons by sources. The scattering function $f$ is normalized so that

$$
\int f\left(\mathbf{\Omega} \cdot \mathbf{\Omega}^{\prime}\right) d \mathbf{\Omega}^{\prime}=1 .
$$

Further simplifying assumptions are those of isotropic scattering and plane symmetry; i.e., $f=1$ and $\psi$ depends only on the position coordinate $x$ and the direction coordinate $\Omega_{x}=\mu$. This reduces Eq. (1) to

$$
\frac{\partial \psi}{\partial t}+\mu v \frac{\partial \psi}{\partial x}+v \sigma \psi=\frac{c}{2} v \sigma \int_{-1}^{1} \psi\left(x, \mu^{\prime}\right) d \mu^{\prime}+Q(x, \mu, t)
$$

Introducing the optical thickness by

$$
x^{\prime}=\int \sigma(x) d x
$$

replacing $Q$ by $q$ where

$$
q\left(x^{\prime}, \mu, t\right)=\frac{Q\left[x\left(x^{\prime}\right), \mu, t\right]}{v \sigma}
$$

and dropping primes gives finally

$$
\frac{1}{v \sigma} \frac{\partial \psi}{\partial t}+\frac{\mu \partial \psi}{\partial x}+\psi=\frac{c}{2} \int_{-1}^{1} \psi\left(x, \mu^{\prime}\right) d \mu^{\prime}+q(x, \mu, t) .
$$

In the first part of the paper only time-independent problems are considered. Thus in Section II solutions of the time-independent, homogeneous form of Eq. (3) are discussed for regions where $c$ is constant.

As an application the Green's function for a uniform infinite medium is constructed in Section III. A rather surprising partial range completeness relation is then proved. A by-product of this is the solution of a generalization of the classical Milne problem. In Section $V$ the application of the method to some timedependent problems is indicated. 


\section{ELEMENTARY SOLUTIONS OF THE TIME-INDEPENDENT EQUATION}

We consider a region where $c$ is constant and look for solutions of the homogeneous equation there. These solutions will depend only on local properties. The idea is to construct special solutions appropriate to various boundary conditions in terms of superpositions of the elementary solutions.

The transport equation is simply

$$
\mu \frac{\partial \psi}{\partial x}+\psi=\frac{c}{2} \int_{-1}^{1} \psi\left(x, \mu^{\prime}\right) d \mu^{\prime}
$$

Translational invariance suggests trying

$$
\psi=e^{-x / \nu} \phi_{\nu}(\mu)
$$

With this assumption, Eq. (4) becomes

$$
(1-\mu / \nu) \phi_{z}(\mu)=\frac{c}{2} \int_{-1}^{1} \phi_{\nu}\left(\mu^{\prime}\right) d \mu^{\prime} .
$$

It is very convenient to normalize so that

$$
\int_{-1}^{1} \phi_{\nu}\left(\mu^{\prime}\right) d \mu^{\prime}=1
$$

Then the above becomes

$$
(\nu-\mu) \phi_{\nu}(\mu)=\frac{c}{2} \nu
$$

From this point the conventional argument runs as follows: Solving Eq. (8) gives

$$
\phi_{\nu}(\mu)=\frac{c}{2} \frac{\nu}{\nu-\mu}
$$

Inserting this result into Eq. (7) yields the condition

$$
1=c \nu \tanh ^{-1} 1 / \nu \text {. }
$$

There are, as is well known, two roots of the equation. Here they will be denoted by $\pm \nu_{o}$. (We note that for all $c,\left|\operatorname{Re} 1 / \nu_{o}\right|<1$ and $\nu_{o}$ is purely imaginary for $c>1$.) The argument has given the usual solutions of the homogeneous transport equation. However, there are others. Thus, Eq. (9) does not strictly follow from Eq. (8). If $\phi_{\nu}(\mu)$ can be a distribution, it can contain a term proportional to $\delta(\nu-\mu)$. Thus from Eq. (8) one can only conclude that

$$
\phi_{\nu}(\mu)=\frac{c}{2} P \frac{\nu}{\nu-\mu}+\lambda(\nu) \delta(\mu-\nu)
$$


where $P$ indicates that principal values are to be understood when integrating an expression involving $\phi_{\nu}(\mu)$. The number $\lambda(\nu)$ is to be determined by condition $(7)$, i.e.,

$$
\int_{-1}^{1} \phi_{\nu}(\mu) d \mu=1=\frac{c}{2} \nu P \int_{-1}^{1} \frac{d \mu}{\nu-\mu}+\lambda(\nu) \int_{-1}^{1} \delta(\mu-\nu) d \nu
$$

There are two pssibilities:

(a) $\nu$ is not real and between -1 and +1 . The second term on the right of (12) is zero and the condition is

$$
1=\frac{c}{2} \nu \int_{-1}^{1} \frac{d \mu}{\nu-\mu}
$$

(The $P$ has been dropped since the denominator does not vanish in the range of integration.) The two roots $\pm \nu_{v}$ occur. With the normalization (7) the corresponding solutions of the transport equation are

$$
\psi_{o \pm}(x, \mu)=\phi_{a \pm}(\mu) e^{\mp x / v_{o}},
$$

where

$$
\phi_{o \pm}(\mu)=\frac{c}{2} \frac{\nu_{o}}{\nu_{o} \mp \mu}
$$

(b) $\nu$ is real and lies between -1 and +1 . The normalization condition is then

$$
1=\frac{c}{2} \nu P \int_{-1}^{1} \frac{d \mu}{\nu-\mu}+\lambda(\nu) .
$$

Clearly $\lambda(\nu)$ can always be chosen so that this is satisfied. Hence there are solutions for all real $\nu$ such that $-1 \leqq \nu \leqq 1$. These are

$$
\psi_{\nu}(x, \mu)=\phi_{\nu}(\mu) e^{-x / \nu},
$$

where

$$
\phi_{\nu}(\mu)=\frac{c \nu}{2} P \frac{1}{\nu-\mu}+\lambda(\nu) \delta(\mu-\nu) .
$$

Here $\lambda(\nu)$ is defined by (14) or, explicitly,

$$
\lambda(\nu)=1-c \nu \tanh ^{-1} \nu .
$$

To summarize: There are two discrete solutions given by Eqs. (13) and a continuum of solutions given by Eqs. (15).

The usefulness of these functions arises from the facts that they are both orthogonal and complete. This can be stated in the form of two theorems. 
Theorem I:

$$
\int_{-1}^{1} \mu \phi_{\nu^{\prime}}(\mu) \phi_{\nu}(\mu)=0, \quad \nu \neq \nu^{\prime}
$$

Proof: $\phi_{v}$ and $\phi_{v^{\prime}}$ satisfy the equations

$$
\begin{aligned}
(1-\mu / \nu) \phi_{\nu}(\mu) & =\frac{c}{2} \int_{-1}^{1} \phi_{\nu}\left(\mu^{\prime}\right) d \mu^{\prime}, \\
\left(1-\mu / \nu^{\prime}\right) \phi_{\nu^{\prime}}(\mu) & =\frac{c}{2} \int_{-1}^{1} \phi_{\nu^{\prime}}\left(\mu^{\prime \prime}\right) d \mu^{\prime \prime} .
\end{aligned}
$$

Multiplying the first of these by $\phi_{\nu^{\prime}}(\mu)$, the second by $\phi_{\nu}(\mu)$, subtracting, and integrating, gives

$$
\left(\frac{1}{\nu^{\prime}}-\frac{1}{\nu}\right) \int_{-1}^{1} \mu \phi_{\nu^{\prime}}(\mu) \phi_{\nu}(\mu) d \mu=0 .
$$

From this the theorem follows.

Since the explicit forms of the functions have been found, the orthogonality integral for $\nu=\nu^{\prime}$ can be readily computed. The results are

$$
\int_{-1}^{1} \mu \phi_{o \pm}^{2}(\mu) d \mu=N_{o \pm}= \pm \frac{c}{2} v_{o}^{3}\left(\frac{c}{v_{o}^{2}-1}-1\right)
$$

and

$$
\int_{-1}^{1} \mu \phi_{p^{\prime}}(\mu) \phi_{v}(\mu) d \mu=N_{\nu} \delta\left(\nu-\nu^{\prime}\right)
$$

with

$$
N_{\nu}=\nu\left[\lambda^{2}(\nu)+\frac{\pi^{2} c^{2}}{4} \nu^{2}\right]
$$

Theorem II:

The functions $\phi_{0 \pm}$ and $\phi_{\nu}(-1 \leqq \nu \leqq 1)$ are complete for functions $\psi(\mu)$ defined in the interval $-1 \leqq \mu \leqq 11^{1}$

The truth of the theorem is suggested by the degenerate case $c=0$. Then $\phi_{\nu}(\mu)=\delta(\nu-\mu)$. These "functions" are obviously "complete." It is worth noting that the proof is constructive in that a method for finding the expansion coefficients is given. Actually the orthogonality relation does this anyway. However, in the general case treated in Section IV it will be seen that an orthogonality relation is not available.

1 The necessary restrictions on $\psi(\mu)$ appear to be very weak and satisfied by all functions of physical interesi. In purticular, the proof seems to apply if $\psi(\mu)$ involves delta functions. 
Proof: I $\mathrm{l}$ is to be shown that one can express $\psi(\mu)$ in the form

$$
\psi(\mu)=a_{o+} \phi_{o+}(\mu)+a_{o-} \phi_{0-}(\mu)+\int_{-1}^{1} A(\nu) \phi_{\nu}(\mu) d \nu .
$$

If the expansion is possible, the coefficients are readily found using Theorem $I$. In particular, it follows from Eqs. (19) and (20) that

$$
a_{o \pm}=\frac{1}{N_{o \pm}} \int_{-1}^{1} \mu \phi_{o \pm}(\mu) \psi(\mu) d \mu .
$$

Hence it is sufficient to show that given any $\psi(\mu)$ the function

$$
\psi^{\prime}(\mu)=\psi(\mu)-a_{o+} \phi_{o+}(\mu)-a_{0-\phi_{0-}}(\mu)
$$

[with $a_{0 \pm}$ give by (24)] can be written as

$$
\psi^{\prime}(\mu)=\int_{-1}^{1} A(\nu) \phi_{\nu}(\mu) d \nu
$$

With (15b) this becomes

$$
\psi^{\prime}(\mu)=\lambda(\mu) A(\mu)+\frac{c}{2} P \int_{-1}^{1} \frac{\mu^{\prime} A\left(\mu^{\prime}\right) d u^{\prime}}{\mu^{\prime}-\mu} .
$$

To prove completeness the existence of a solution of this singular integral equation must be demonstrated. Such problems have been extensively treated. The essential point is to relate the functions which occur to the boundary values of functions of a complex variable. The properties which can be ascribed to these functions serve to determine them completely. In the present case not all the available apparatus is needed. ${ }^{2}$ We follow a shortcut suggested by Van Kampen (1).

Suppose a solution $A(\nu)$ exists. Introduce a function $N(z)$ of the complex variable $z$ by

$$
N(z)=\frac{1}{2 \pi i} \int_{-1}^{1} \frac{c}{2} \frac{\nu A(\nu) d \nu}{\nu-z}
$$

This function vanishes as $|z| \rightarrow \infty$ and is analytic in the complex plane cut from -1 to +1 . For the limits as $z$ approaches the cut from above $(+)$ and below $(-)$, we have

$$
N^{ \pm}(\mu)=\frac{1}{2 \pi i} P \int_{-1}^{1} \frac{c}{2} \frac{\nu A(\nu) d \nu}{\nu-\mu} \pm \frac{1}{2} \frac{c}{2} \mu A(\mu) .
$$

Hence

$$
N^{+}+N^{-}=\frac{1}{\pi i} P \int_{-1}^{1} \frac{c}{2} \frac{\nu A(\nu) d \nu}{\nu-\mu},
$$

2 In Appendix A an alternate approach using the general theory is shown. 
and

$$
N^{+}-N^{-}=\frac{c}{2} \mu A(\mu)
$$

In terms of $N^{ \pm}$the integral equation (27) becomes

$$
\frac{c}{2} \mu \psi^{\prime}(\mu)=\left[\lambda(\mu)+i \frac{\pi}{2} c \mu\right] N^{+}(\mu)-\left[\lambda(\mu)-i \frac{\pi c \mu}{2}\right] N^{-}(\mu) .
$$

The explicit form for $\lambda(\mu)$ could now be introduced. However, it is more convenient to proceed as follows: Introduce a function $Q(z)$ by

$$
Q(z)=\frac{1}{2 \pi i} \frac{c}{2} z \int_{-1}^{1} \frac{d \mu^{\prime}}{\mu^{\prime}-z} .
$$

$Q(z)$ has similar properties to $N(z)$ except that it tends to $-c / 2 \pi i$ as $z \rightarrow \infty$. In particular

$$
Q^{ \pm}(\mu)=\frac{1}{2 \pi i} \frac{c}{2} \mu P \int_{-1}^{1} \frac{d u^{\prime}}{\mu^{\prime}-\mu} \pm \frac{1}{2} \frac{c}{2} \mu .
$$

Therefore,

$$
Q^{+}-Q^{-}=\frac{c}{2} \mu
$$

and

$$
Q^{+}+Q^{-}=\frac{1}{\pi i} \frac{c}{2} \mu P \int_{-1}^{1} \frac{d u^{\prime}}{\mu^{\prime}-\mu}
$$

In terms of these boundary values one sees that

$$
\lambda(\mu)=1+\pi i\left(Q^{+}+Q^{-}\right)
$$

and

$$
\frac{\pi i c \mu}{2}=\pi i\left(Q^{+}-Q^{-}\right)
$$

Then the integral equation is:

$$
\left[1+2 \pi i Q^{+}(\mu)\right] N^{+}(\mu)-\left[1+2 \pi i Q^{-}(\mu)\right] N(\mu)=\frac{c}{2} \mu \psi^{\prime}(\mu) .
$$

Finally consider the function

$$
F(z)=[1+2 \pi i Q(z)] N(z)-\frac{1}{2 \pi i} \int_{-1}^{1} \frac{c}{2} \frac{\mu^{\prime} \psi^{\prime}\left(\mu^{\prime}\right) d \mu^{\prime}}{\mu^{\prime}-z}
$$


This function is zero at infinity and analytic in the complex plane except perhaps for a branch cut from $-1 \leqq z \leqq 1$. On this cut the discontinuity of $F(z)$ is:

$$
F^{+}(\mu)-F^{-}(\mu)=\left[1+2 \pi i Q^{+}\right] N^{+}-\left[1+2 \pi i Q^{-}\right] N^{-}-\frac{c}{2} \mu \psi^{\prime}(\mu)=0 .
$$

Hence $F(z)$ is analytic everywhere. Since it vanishes at infinity, one concludes

$$
F(z)=0
$$

and thus

$$
N(z)=\frac{1}{[1+2 \pi i Q(z)]} \frac{1}{2 \pi i} \int_{-1}^{1} \frac{c}{2} \frac{\mu^{\prime} \psi^{\prime}\left(\mu^{\prime}\right) d \mu^{\prime}}{\mu^{\prime}-z}
$$

So far it has been assumed that $A(\nu)$ exists. Then the function $N(z)$ is given by (40) and $A(\nu)$ can be determined from (31). Conversely, if $N(z)$ given by (40) has the required analytic properties [see remarks after (28)], then $A(\nu)$ given by (31) exists and satisfies the integral equation. The crucial question is then as to whether $N(z)$ is analytic in the cut plane and vanishes at infinity. The numerator in (40) does have these properties. The denominator is analytic and goes to a constant at infinity. ${ }^{3}$ Hence the question is whether the numerator vanishes at the zeros of the denominator, i.e., is

$$
\frac{1}{2 \pi i} \int_{-1}^{1} \frac{c}{2} \frac{\mu^{\prime} \psi^{\prime}\left(\mu^{\prime}\right) d \mu^{\prime}}{\mu^{\prime}-z}=0
$$

when

$$
1+2 \pi i Q(z)=0 ?
$$

By definition

$$
Q(z)=\frac{1}{2 \pi i} \frac{c}{2} z \int_{-1}^{1} \frac{d \mu^{\prime}}{\mu^{\prime}-z}=\frac{1}{2 \pi i}\left(-c z \tanh ^{-1} z\right)
$$

Hence the condition of Eq. (42) is

$$
1=c z \tanh ^{-1} z
$$

which is just the transcendental equation for the discrete solutions. The points of interest are therefore $\pm \nu_{o}$. Is (41) satisfied at these points? That is, is

$$
-\frac{c}{2} \nu_{o} \int_{-1}^{1} \frac{\mu^{\prime} \psi^{\prime}\left(\mu^{\prime}\right) d \mu^{\prime}}{\mu^{\prime} \mp \nu_{o}}=0 ?
$$

3 The apparent difficulty for the case $c=1$ is illusory. A careful analysis leads to exactly the results expected by taking limits $c \rightarrow 1+$ or $c \rightarrow 1-$. 
Comparing with Eq. (13b), it is seen that

$$
\phi_{o \pm}(\mu)=-\frac{c}{2} \frac{\nu_{o}}{\mu^{\prime} \mp \nu_{o}} .
$$

Hence the left side of (44) is

$$
\int_{-1}^{1} \mu^{\prime} \phi_{0 \pm}\left(\mu^{\prime}\right) \psi^{\prime}\left(\mu^{\prime}\right) d \mu^{\prime}
$$

Using Eqs. (24) and (25), it follows that this expression is

$$
\begin{gathered}
\int_{-1}^{1} \mu^{\prime} \phi_{o \pm}\left(\mu^{\prime}\right) \psi\left(\mu^{\prime}\right) d \mu^{\prime}-\int_{-1}^{1} \mu^{\prime} \phi_{o \pm}\left(\mu^{\prime}\right) \phi_{o+}\left(\mu^{\prime}\right) d \mu^{\prime} \frac{1}{N_{o+}} \int_{-1}^{1} \mu \phi_{o+}(\mu) \psi(\mu) d \mu \\
-\int_{-1}^{1} \mu^{\prime} \phi_{o \pm}\left(\mu^{\prime}\right) \phi_{o-}\left(\mu^{\prime}\right) d \mu^{\prime} \frac{1}{N_{o-}} \int_{-1}^{1} \mu \phi_{o-}(\mu) \psi(\mu) d \mu \\
=\int_{-1}^{1} \mu^{\prime} \phi_{o \pm}\left(\mu^{\prime}\right) \psi\left(\mu^{\prime}\right) d \mu^{\prime}-\int_{-1}^{1} \mu \phi_{o \pm}(\mu) \psi(\mu) d \mu=0
\end{gathered}
$$

(Here the orthogonality and normalization properties of the $\phi_{o \pm}$ have been used.) Thus, the question of Eq. (44) is answered affirmatively. $N(z)$ has the desired properties. $A(\nu)$ exists [and can be found using Eq. (31)].

\section{GREEN'S FUNCTION FOR A UNIFORM INFINITE MEDIUM}

As an illustration of the applicability of the results of the preceding section, the Green's function for the transport equation will be constructed. To be definite $c<1$ is assumed here. ${ }^{4}$ The Green's function $\psi_{g}$ satisfies the equation

$$
\mu \frac{\partial \psi_{g}}{\partial x}+\psi_{g}=\frac{c}{2} \int_{-1}^{1} \psi_{g}\left(x, \mu^{\prime}\right) d \mu^{\prime}+\frac{\delta(x)}{4 \pi} \delta\left(\mu-\mu_{o}\right)
$$

Integrating across the plane $x=0$ shows that $\psi_{g}$ satisfies the homogeneous equation for $x \neq 0$ and the jump condition

$$
\mu\left[\psi_{g}\left(0^{+}, \mu\right)-\psi_{g}\left(0^{-}, \mu\right)\right]=\frac{1}{4 \pi} \delta\left(\mu-\mu_{0}\right)
$$

Let us look for the solution $\psi_{g}$ which vanishes as $|x| \rightarrow \infty$. It is sufficient to expand $\psi_{g}$ in the form

$$
\psi_{g}=a_{0+} \psi_{o+}(x, \mu)+\int_{0}^{1} A(\nu) \psi_{\nu}(x, \mu) d \nu, \quad x>0,
$$

- The cases $c>1$ or $c=1$ can be treated analogously. 
or

$$
\psi_{g}=-a_{o-} \psi_{o-}(x, \mu)-\int_{-1}^{0} A(\nu) \psi_{\nu}(x, \mu) d \nu, \quad x<0 .
$$

Condition (48) then gives an integral equation to determine the expansion coefficients. It is

$$
\frac{\delta\left(\mu-\mu_{o}\right)}{4 \pi \mu}=a_{o+} \phi_{o+}(\mu)+a_{o-} \phi_{o-}(\mu)+\int_{-1}^{1} A(\nu) \phi_{\nu}(\mu) d \nu .
$$

The solution obtained using the orthogonality relations is

$$
a_{o \pm}=\frac{1}{N_{o \pm}} \int_{-1}^{1} \frac{\mu \phi_{o \pm}(\mu) \delta\left(\mu-\mu_{o}\right) d \mu}{4 \pi \mu}=\frac{1}{4 \pi} \frac{\phi_{o \pm}\left(\mu_{o}\right)}{N_{o \pm}},
$$

and

$$
A(\nu)=\frac{1}{4 \pi} \frac{\phi_{\nu}\left(\mu_{o}\right)}{N_{\nu}} .
$$

Hence $\psi_{\theta}$ can be written in the typical normal mode expansion

$$
\begin{aligned}
\psi_{g} & =\frac{1}{4 \pi}\left[\frac{\phi_{0+}\left(\mu_{o}\right) \phi_{o+}(\mu) e^{-x / \nu_{o}}}{N_{o+}}+\int_{0}^{1} \frac{\phi_{\nu}\left(\mu_{o}\right) \phi_{\nu}(\mu) e^{-x / \nu}}{N_{\nu}} d \nu\right] . & & (x>0) \\
& =-\frac{1}{4 \pi}\left[\frac{\phi_{o-}\left(\mu_{o}\right) \phi_{o-}(\mu) e^{x / \nu_{o}}}{N_{o-}}+\int_{-1}^{0} \frac{\phi_{\nu}\left(\mu_{o}\right) \phi_{\nu}(\mu) e^{-x / \nu}}{N_{\nu}} d \nu\right] . & & (x<0)
\end{aligned}
$$

Particularly simple results follow for the angular distributions and densities due to an isotropic plane source. For the angular density $\psi_{o}$ we have

$$
\begin{aligned}
\psi_{o}(x, \mu) & =\int_{-1}^{1} \psi_{0}\left(x, \mu ; \mu_{o}\right) d \mu_{o} \\
& =\frac{1}{4 \pi}\left[\frac{\phi_{o+}(\mu) e^{-|x| / \nu_{o}}}{N_{o+}}+\int_{0}^{1} \frac{\phi_{\nu}(\mu) e^{-|x| / \nu}}{N_{\nu}} d \nu\right],
\end{aligned}
$$

where the normalization of Eq. (7) has been used. To find the neutron density it is only necessary to integrate (53) over all $\mu$. This yields

$$
\rho_{o}(x)=\frac{1}{4 \pi}\left[\frac{e^{-|x| / \nu_{o}}}{N_{o+}}+\int_{0}^{1} \frac{e^{-|x| / \nu}}{N_{\nu}} d \nu\right] .
$$

It is worth noting that the method is well suited to finding the asymptotic behavior of solutions. This is clearly given by the two discrete eigenfunctions. The coefficients are readily obtained.

\section{PARTIAL RANGE COMPLETENESS}

The elementary solutions found in Section II have a much more general completeness property than is indicated by Theorem II. This can be stated as: 


\section{THEOREM III:}

Let $\alpha$ and $\beta$ be two real numbers such that $-1 \leqq \alpha<\beta \leqq 1$. Then

(a) If $\alpha \neq-1$ and $\beta \neq+1$, the functions $\phi_{\nu}(\mu)(\alpha \leqq \nu \leqq \beta)$ are complete for functions $\psi(\mu)$ defined in the interval $\alpha \leqq \mu \leqq \beta$.

(b) If $\alpha=-1(\beta=+1)$, the set $\phi_{\nu}(\alpha \leqq \nu \leqq \beta)$ is complete when supplemented by $\phi_{o-}\left(\phi_{o+}\right)$.

It is believed that the theorem is true under quite weak restrictions on the function $\psi(\mu)$. However, the result is peculiar enough that it seems worthwhile to give a rigorous mathematical proof. Thus, to be explicit, it is assumed that $\psi(\mu)$ satisfies a Hölder condition in the open interval $\alpha<\mu<\beta$ and is bounded by (constant) $/|\mu-\alpha|^{\gamma}$ or (constant) $/|\mu-\beta|^{\gamma}$ with $\gamma<1$ at the endpoints. The proof consists of tracing through in detail the solution of singular integral equations given by Muskhelishvili ( $\mathscr{2}$ ). In essence we ask for the conditions under which the integral equation implied by the possibility of expansion has a solution. It will be seen that either there is a solution or the conditions can be satisfied by appending the functions $\phi_{o-}$, or $\phi_{o+}$, or both.

Proof: If $\psi(\mu)$ is an arbitary function (subject to the above conditions) defined in the interval $-1 \leqq \alpha \leqq \mu \leqq \beta \leqq 1$, it is to be shown that

$$
\psi(\mu)=\int_{\alpha}^{\beta} A(\nu) \phi_{\nu}(\mu) d \nu, \quad(\alpha \leqq \mu \leqq \beta),
$$

i.e., a solution of the integral equation

$$
\psi(\mu)=\lambda(\mu) A(\mu)+\frac{c}{2} P \int_{\alpha}^{\beta} \frac{\nu A(\nu) d \nu}{\nu-\mu}
$$

exists. Suppose there is such an $A(\nu)$ with properties similar to those of $\psi(\mu)$. Introduce a function of the complex variable $z$ by

$$
N(z)=\frac{1}{2 \pi i} \int_{\alpha}^{\beta} \frac{c}{2} \frac{\nu A(\nu) d \nu}{\nu-z}
$$

This function has the properties:

(1) It is analytic in the complex plane with a cut from $\alpha$ to $\beta$.

(2) $N(z) \sim 1 / z$ as $z \rightarrow \infty$.

(3) $N(z)$ is bounded by $C_{1} /|\alpha-z|^{\gamma}$ and $C_{2} /|\beta-z|^{\gamma}$ with $\gamma<1$ as $z \rightarrow \alpha$ and $\beta$, respectively.

Further,

$$
N^{+}+N^{-}=\frac{1}{\pi i} P \int_{\alpha}^{\beta} \frac{c}{2} \frac{\nu A(\nu)}{\nu-\mu} d \nu
$$

and

$$
N^{+}-N^{+}=\frac{c}{2} \nu A(\nu)
$$


The integral equation (56) is:

$$
\frac{c}{2} \mu \psi(\mu)=\left[\lambda(\mu)+\frac{i \pi}{2} c \mu\right] N^{+}(\mu)-\left[\lambda(\mu)-\frac{i \pi}{2} c \mu\right] N^{-}(\mu),
$$

or

$$
G(\mu) N^{+}(\mu)-N(\mu)=\frac{c}{2} \frac{\mu \psi(\mu)}{\lambda(\mu)-(i \pi / 2) c \mu},
$$

where

$$
G(\mu)=\frac{\lambda(\mu)+(i \pi / 2) c \mu}{\lambda(\mu)-(i \pi / 2) c \mu}
$$

Suppose $G(\mu)=X^{+}(\mu) / X^{-}(\mu)$ where:

(1) $X(z)$ is analytic in the cut plane.

(2) $X(z)$ is nonvanishing in the cut plane.

(3) $X(z)$ does not vanish as rapidly as $\alpha-z$ or $\beta-z$ as $z \rightarrow \alpha$ or $\beta$, respectively.

(4) At infinity $X(z)$ behaves in one of the following ways:

(a) $X(z) \rightarrow$ constant (nonzero);

(b) $X(z) \rightarrow z^{-n}$ ( $n$ integral and positive);

(c) $X(z) \rightarrow z^{m}$ ( $m$ integral and positive).

Then

$$
F(z)=X(z) N(z)-\frac{1}{2 \pi i} \int_{\alpha}^{\beta} \frac{\overline{2}}{2} \frac{\mu^{\prime} \psi\left(\mu^{\prime}\right) X\left(\mu^{\prime}\right) d \mu^{\prime}}{\left[\lambda\left(\mu^{\prime}\right)-(i \pi / 2) c \mu^{\prime}\right]\left(\mu^{\prime}-z\right)}
$$

is analytic in the cut plane and continuous across the cut. It has at most removable singularities at $\alpha$ and $\beta$. In cases (a) and (b), $F(z)$ vanishes at infinity. Hence

$$
F(z)=0
$$

and therefore

$$
N(z)=\frac{1}{X(z)} \frac{1}{2 \pi i} \int_{\alpha}^{\beta} \frac{c}{2} \frac{\mu^{\prime} \psi\left(\mu^{\prime}\right) X^{-}\left(\mu^{\prime}\right) d \mu^{\prime}}{\left[\lambda\left(\mu^{\prime}\right)-(i \pi / 2) c \mu^{\prime}\right]\left(\mu^{\prime}-z\right)}
$$

In case (c), $F(z) \sim z^{m-1}$ at infinity. Hence

$$
F(z)=P_{m-1}(z),
$$

where $P_{m-1}(z)$ is an arbitrary polynomial of degree $m-1$. Then

$$
N(z)=\frac{1}{X(z)} \frac{1}{2 \pi i} \int_{\alpha}^{\beta} \frac{c}{2} \frac{\mu^{\prime} \psi\left(\mu^{\prime}\right) X^{-}\left(\mu^{\prime}\right) d \mu^{\prime}}{\left[\lambda\left(\mu^{\prime}\right)-(i \pi / 2) c \mu^{\prime}\right]\left(\mu^{\prime}-z\right)}+\frac{P_{m-1}(z)}{X(z)} .
$$


Thus, if a solution $A(\nu)$ exists and if there is an appropriate $X(z)$, the function $N(z)$ is given by Eqs. (65). Conversely, given $X(z)$ we consider the function $N(z)$ defined by Eqs. (65). We can then find the solution $A(\nu)$ using Eq. (59)provided the conditions listed after Eq. (57) are fulfilled.

In cases (a) and (c) these are satisfied for all $\psi(\mu)$. Hence the solutions exist. [It is unique in case (a) but not in case (c)]. In case (b) the condition (2) is the only one not automatically satisfied. A solution (unique) exists only if

$$
\int_{\alpha}^{\beta} \mu^{l}\left[\frac{\mu \psi(\mu) X^{-}(\mu)}{\lambda(\mu)-(i \pi / 2) c \mu}\right] d \mu=0, \quad l=0,1,2, \cdots, n-1 .
$$

We turn to the construction of $X(z)$. Taking logarithms of both sides of Eq. (62) gives

$$
\ln X^{+}-\ln X^{-}=G(\mu)
$$

If $\Gamma(z)$ be defined by

$$
\Gamma(z)=\frac{1}{2 \pi i} \int_{\alpha}^{\beta} \frac{\ln G\left(\mu^{\prime}\right) d \mu^{\prime}}{\mu^{\prime}-z}
$$

then

$$
X_{o}(z)=e^{\Gamma(z)}
$$

satisfies Eq. (67). Moreover, since $\Gamma(z)$ is analytic in the cut plane and vanishes at infinity, $X_{o}(z)$ is a satisfactory $X(z)$ [case (a)] provided the behavior in the neighborhood of the points $\alpha$ and $\beta$ [condition (3)] is all right. Let us examine this behavior.

For $z \approx \alpha$,

$$
\Gamma(z) \approx \frac{1}{2 \pi i} \ln G(\alpha) \int_{\alpha} \frac{d \mu^{\prime}}{\mu^{\prime}-z}=-\frac{1}{2 \pi i} \ln G(\alpha) \ln (\alpha-z),
$$

Similarly, for $z$ near $\beta$

$$
\Gamma(z) \approx \frac{1}{2 \pi i} \ln G(\beta) \ln (\beta-z) .
$$

Now from Eq. (62) it follows that

$$
\frac{1}{2 \pi i} \ln G(\mu)=\frac{1}{\pi} \theta(\mu),
$$

where

$$
\theta(\mu)=\arg \left[\lambda(\mu)+\frac{\pi i c \mu}{2}\right]=\arg \left[1-c \mu \tanh ^{-1} \mu+\frac{\pi i c \mu}{2}\right]
$$


Thus, ncar $\alpha$ and $\beta$

$$
X_{o}(z) \approx(\alpha-z)^{-\theta(\alpha) / \pi}
$$

or

$$
X_{o}(z) \approx(\beta-z)^{\theta(\beta) / \pi} .
$$

When $\mu$ varies between -1 and +1 the function $\theta(\mu)$ varies between $-\pi$ and $+\pi \cdot[\theta(0)=0$ and $\theta(-\mu)=-\theta(\mu)$. $]$

$$
-1 \leqq \frac{\theta(\mu)}{\pi} \leqq 1
$$

Hence there are integers $l(\alpha)$ and $k(\beta)$ which are either $+1,0$, or -1 such that

$$
1>l(\alpha)-\frac{\theta(\alpha)}{\pi} \geqq 0,
$$

and

$$
1>k(\beta)+\frac{\theta(\beta)}{\pi} \geqq 0 .
$$

These numbers are given in Table $\mathrm{I}$.

An appropriate $X(z)$ is

$$
X(z)=(\alpha-z)^{l(\alpha)}(\beta-z)^{k(\beta)} X_{o}(z) .
$$

At infinity $X(z) \sim z^{N(\alpha, \beta)}$, where

$$
N(\alpha, \beta)=l(\alpha)+l(\beta) .
$$

The values of $N$ and the class of $X(z)$ (and the corresponding $n$ and $m$ ) are given in Table I. From this we note that for all possible $\alpha$ and $\beta$ the $X(z)$ lie in one of the three classes (a), (b), or (c). If neither $\alpha=-1$ nor $\beta=+1$ the class (b) does not occur. Thus in those cases the integral equation possesses a solution

TABLE I

Classification of the Functions $X(z)$

\begin{tabular}{rrrrrl}
\hline$\alpha$ & $l(\alpha)$ & $\beta$ & $k(\beta)$ & $N(\alpha, \beta)$ & Class of $X(z)$ \\
\hline-1 & -1 & +1 & -1 & -2 & $b(n=2)$ \\
-1 & -1 & $0 \leqq \beta<1$ & 0 & -1 & $b(n=1)$ \\
-1 & -1 & $-1<\beta<0$ & +1 & 0 & $a$ \\
$-1<\alpha \leqq 0$ & 0 & +1 & -1 & -1 & $b(n=1)$ \\
$-1<\alpha \leqq 0$ & 0 & $0 \leqq \beta<1$ & 0 & 0 & $a$ \\
$-1<\alpha \leqq 0$ & 0 & $\alpha \leqq \beta<0$ & +1 & +1 & $c(m=1)$ \\
$0<\alpha<1$ & +1 & +1 & -1 & 0 & $a$ \\
$0<\alpha<1$ & +1 & $\alpha<\beta<1$ & 0 & +1 & $c(m=+1)$ \\
\hline
\end{tabular}


and the expansion is possible. ${ }^{5}$ The cases where $X(z)$ is a member of class (b) require special attention. Consider $\alpha=-1, \beta=+1$. Then $n=2$ and a solution exists only if

$$
\int_{-1}^{1} \mu^{l} \frac{\left[\mu \psi(\mu) X^{-}(\mu)\right]}{\lambda(\mu)-(i \pi / 2) c \mu} d \mu=0, \quad l=0,1 .
$$

These will certainly not be satisfied in general. But we can modify the procedure. Instead of $\psi(\mu)$, consider the function

$$
\psi^{\prime}(\mu)=\psi(\mu)-a_{o+} \phi_{o+}(\mu)-a_{o-} \phi_{o-}(\mu) .
$$

Here $a_{o \pm}$ are unknown constants. The condition that $\psi^{\prime}(\mu)$ can be expanded in terms of the $\phi_{\nu}(\mu)$ is then that the two conditions of Eq. (79) be satisfied with $\psi(\mu)$ replaced by $\psi^{\prime}(\mu)$. These conditions are then used to determine $a_{o \pm}$. Thus we always have the possibility of the expansion

$$
\psi(\mu)=a_{o+} \phi_{o+}(\mu)+a_{o-} \phi_{o-}(\mu)+\int_{-1}^{1} A(\nu) \phi_{\nu}(\mu) d \nu .
$$

Moreover, we have a procedure to determine the expansion coefficients.

A second special case of particular interest is $\alpha=0, \beta=1$. Then, from Table I, $X(z)$ is a member of class (b) with $n=+1$. The expansion is possible only if

$$
\int_{0}^{1} \frac{\left[\mu \psi(\mu) X^{-}(\mu)\right]}{\lambda(\mu)-(i \pi / 2) c \mu} d \mu=0 .
$$

Again the procedure is modified. Consider $\psi^{\prime}(\mu)=\psi(\mu)-a_{o+} \phi_{o+}(\mu)$. This can be expanded in terms of $\phi_{\nu}(\mu)(0 \leqq \nu \leqq 1)$ provided Eq. (80) with $\psi(\mu)$ replaced by $\psi^{\prime}(\mu)$ is satisfied. This is accomplished by suitable choice of $a_{o+}$, i.e.,

$$
a_{o+}=\frac{\int_{-0}^{1} \gamma(\mu) \psi(\mu) d \mu}{M_{o+}},
$$

where

$$
\gamma(\mu)=\frac{\mu X^{-}(\mu)}{[\lambda(\mu)-(i \pi / 2) c \mu]},
$$

and $^{6}$

$$
M_{o+}=\int_{0}^{1} \gamma(\mu) \phi_{o+}(\mu) d \mu .
$$

The other special case of class (b) can be treated in similar fashion.

${ }^{5}$ It is interesting to note that for $\alpha, \beta$ both on the same side of the origin $X(z)$ belongs to class $c$. The expansion coefficients are then not unique.

${ }^{6}$ Of course $M_{0+}$ must not vanish. This is proved in Appendix B. 


\section{APPLICATIONS OF THE HALF-RANGE \\ COMPLETENESS RELATIONS}

The first of our applications is to the albedo problem. A plane parallel beam is incident at $x=0$ on the half space $0 \leqq x<\infty$. The problem is to find a solution $\Psi_{a}$ of Eq. (4) in this region subject to the conditions:

$$
\begin{aligned}
& \text { (a) } \Psi_{a}(0, \mu)=\delta\left(\mu-\mu_{o}\right) \quad \mu, \mu_{o}>0, \\
& \text { (b) } \lim _{x \rightarrow \infty} \Psi_{a}(x, \mu)=0 .
\end{aligned}
$$

A general solution of (4) subject to condition (b) is

$$
\Psi_{a}(x, \mu)=a_{o+} \psi_{o+}(x, \mu)+\int_{0}^{1} A(\nu) \psi_{\nu}(x, \mu) d \nu
$$

Condition (a) gives the equation

$$
\delta\left(\mu-\mu_{o}\right)=a_{o+} \phi_{o+}(\mu)+\int_{0}^{1} A(\nu) \phi_{\nu}(\mu) d \nu, \quad(\mu \geqq 0) .
$$

The solution $a_{o+}, A(\nu)$ is given by the completeness proof above. In particular the asymptotic form is determined by $a_{o+}$ where

$$
a_{o+}=\frac{\gamma\left(\mu_{o}\right)}{M_{o+}} \text {. }
$$

Secondly, we consider the Milne problem. A solution $\Psi_{o}$ of Eq. (4) is required in the region $0 \leqq x<\infty$ subject to the conditions

(a) $\Psi_{o}(0, \mu)=0, \quad \mu \geqq 0$,

(b) $\lim _{x \rightarrow \infty} \Psi_{o}(x, \mu)=\psi_{o-}(x, \mu)$.

The general solution of Eq. (4) subject to condition (b) can be written as

$$
\Psi_{o}(x, \mu)=\psi_{o-}(x, \mu)+a_{o+} \psi_{o+}(x, \mu)+\int_{0}^{1} A(\nu) \psi_{\nu}(x, \mu) d \nu .
$$

Condition (a) then requires that

$$
-\phi_{v-}(\mu)=a_{v+} \phi_{o+}(\mu)+\int_{0}^{1} A(\nu) \phi_{\nu}(\mu) d \nu .
$$

Again the solution has been found above. All that is needed is to put $\psi(\mu)=$ $-\phi_{\sigma_{-}}(\mu)$ in the formulas of Section IV. The important coefficient $a_{o+}$ is given by

$$
a_{o+}=-\frac{\int_{0}^{1} \gamma(\mu) \phi_{o-}(\mu)}{M_{o+}} .
$$


A generalization of the Milne problem suggests itself. We ask for a solution $\Psi_{\nu}(x, \mu)$ of Eq. (4) subject to condition (a) and

$\left(b^{\prime}\right) \lim _{x \rightarrow \infty} \Psi_{\nu}(x, \mu)=\psi_{\nu}(x, \mu), \quad(-1 \leqq \nu \leqq 0)$.

The solution of this Milne problem is obviously obtained just as that of the original one. Thus, instead of a single solution of the half-space problem with zero incoming flux, we have one discrete solution plus a continuum of solutions. This set of solutions determines the Green's function for a half space. Thus suppose it is required to solve the equation

$$
\mu \frac{\partial}{\partial x} \Psi_{g}+\Psi_{g}=\frac{c}{2} \int_{-1}^{1} \Psi_{g}\left(x, \mu^{\prime}\right) d \mu^{\prime}+\frac{\delta\left(x-x_{o}\right)}{4 \pi} \delta\left(\mu-\mu_{o}\right)
$$

in the region $0 \leqq x<\infty$ subject to the boundary conditions

$$
\Psi_{g}(0, \mu)=0 \quad \mu \geqq 0,
$$

and

$$
\lim _{x \rightarrow \infty} \Psi_{g}(x, \mu)=0 .
$$

We write down the solution and then check the properties.

$$
\begin{aligned}
\Psi_{o}=\psi_{o}\left(x-x_{o}\right)-\frac{1}{4 \pi}\left\{\frac{\phi_{o-}\left(\mu_{o}\right)}{N_{o-}}\right. & {\left[\Psi_{o}(x, \mu)-\psi_{o-}(x, \mu)\right] e^{-x_{o} / \nu_{o}} } \\
& \left.+\int_{-1}^{0} \frac{\phi_{\nu}\left(\mu_{o}\right)}{N \nu}\left[\Psi_{\nu}(x, \mu)-\psi_{\nu}(x, \mu)\right] e^{x_{o} / \nu} d \nu\right\} .
\end{aligned}
$$

$\psi_{0}$ is the infinite medium Green's function given by Eq. (52) and hence satisfies Eq. (90). The remainder is a solution of the homogeneous equation. Hence Eq. (90) is satisfied. Condition (90b) is fulfilled since $\Psi_{o}(x, \mu)-\psi_{o-}(x, \mu)$ and $\Psi_{\nu}(x, \mu)-\psi_{\nu}(x, \mu)$ are combinations of decreasing exponentials. Finally, at $x=0$ the expression becomes

$$
\Psi_{g}(0, \mu)=-\frac{1}{4 \pi} \frac{\phi_{o-}\left(\mu_{o}\right)}{N_{o-}} \Psi_{o-}(0, \mu) e^{-x_{o} / \nu_{o}}+\int_{-1}^{0} \frac{\phi_{\nu}\left(\mu_{o}\right)}{N_{\nu}} \Psi_{\nu}(0, \mu) e^{x_{o} / \nu} d \nu
$$

and each term vanishes for $\mu \geqq 0$.

\section{TIME-DEPENDENT PROBLEMS}

The method is readily generalizable to time-dependent problems. Consider a region where $\sigma$ and $c$ are constant. Choose units so that $v=\sigma=1$. In this region the homogeneous form of the transport equation (3) is:

$$
\frac{\partial \psi}{\partial t}+\mu \frac{\partial \psi}{\partial x}+\psi=\frac{c}{2} \int_{-1}^{1} \psi\left(x, \mu^{\prime}\right) d \mu^{\prime}
$$


Look for solutions of the form

$$
\psi(x, \mu)=e^{i k x} e^{-(1+i \alpha k) t} \phi_{\alpha, k}(\mu) .
$$

( $k$ is any fixed real number. The permissible values of $\alpha$ are to be determined.) With this assumption Eq. (93) becomes

$$
(\alpha-\mu) \phi_{\alpha, k}(\mu)=\frac{i c}{2 k} \int_{-1}^{1} \phi_{\alpha, k}\left(\mu^{\prime}\right) d \mu^{\prime} .
$$

Since a generalization of this eigenvalue problem has been discussed elsewhere $(3)$, it is sufficient to state the conclusions.

For each $k$ there is a continuum of solutions $\phi_{\alpha, k}(\mu)$ with $-1 \leqq \alpha \leqq 1$. These are

$$
\phi_{\alpha, k}(\mu)=\frac{i c}{2 k} P \frac{1}{\alpha-\mu}+\lambda(\alpha) \delta(\mu-\alpha)
$$

where

$$
\lambda(\alpha)=1-\frac{i c}{k} \tanh ^{-1} \alpha
$$

These solutions are orthogonal in that

$$
\int_{-1}^{1} \phi_{\alpha, k}(\mu) \phi_{\alpha^{\prime}, k}(\mu) d \mu=N_{\alpha, k} \delta\left(\alpha-\alpha^{\prime}\right),
$$

with

$$
N_{\alpha, k}=\lambda^{2}(\alpha)-\frac{c^{2} \pi^{2}}{4 k^{2}}
$$

In addition, for $|k|<c \pi / 2$ there is a discrete solution

$$
\phi_{o, k}(\mu)=\frac{i c}{2 k} \frac{1}{\alpha_{n}-\mu}
$$

where

$$
1=\frac{c}{k} \tanh ^{-1} 1 / \alpha_{o} .
$$

This solution is orthogonal to the continuum solutions, that is,

$$
\int_{-1}^{1} \phi_{o, k}(\mu) \phi_{\alpha, k}(\mu) d \mu=0
$$

and

$$
\int_{-1}^{1} \phi_{o, k}^{2}(\mu) d \mu=\frac{-\lambda c^{2}}{2 k^{2}\left(1-\alpha_{o}^{2}\right)}=N_{o, k}
$$


For any $k$ the enumerated solutions are complete for functions $\psi(\mu)$ defined in the range $-1 \leqq \mu \leqq 1$. They are all normalized so that

$$
\int_{-1}^{1} \phi_{() k}(\mu) d \mu=1
$$

As an application we will solve the initial value problem for a uniform infinite medium. It is sufficient to consider an initial distribution which is

$$
\Psi_{i}(x, \mu ; 0)=\delta\left(x-x_{o}\right) \delta\left(\mu-\mu_{o}\right) .
$$

Expand in the complete set of functions $e^{i k x} \phi_{\alpha, k}(\mu)$, that is,

$$
\Psi_{i}(x, \mu ; 0)=\frac{1}{\sqrt{2 \pi}} \int_{-\infty}^{\infty} d k e^{i k x} \sum_{\alpha} \phi_{\alpha, k}(\mu) A(\alpha, k) .
$$

(Here $\sum_{\alpha}$ means adding the discrete term to an integral over the continuum.) We readily find the expansion coefficients using the orthogonality properties. The distribution at a time $t$ is then [using the time dependence of the eigenfunctions indicated by Eq. (94)]

$$
\Psi_{i}(x, \mu, t)=\frac{e^{-t}}{2 \pi} \int_{-\infty}^{\infty} e^{i k\left(x-x_{0}\right)} d k \sum_{\alpha} \frac{\phi_{\alpha, k}\left(\mu_{0}\right) \phi_{\alpha, k}(\mu)}{N_{\alpha, k}} e^{-i \alpha k t} .
$$

The neutron density $\rho$ due to an initial distribution which is located at $x_{0}$ but uniform in velocity directions is particularly simple. All that is needed is to integrate (107) with respect to $\mu_{o}$ and $\mu$ and remember Eq. (104). The result is

$$
\rho(x, t)=\frac{e^{-t}}{2 \pi} \int_{-\infty}^{\infty} e^{i k\left(x-x_{0}\right)} d k \sum_{\alpha} \frac{e^{-i \alpha k t}}{N_{\alpha, k}} .
$$

Finally, the time-dependent Green's function for a uniform infinite medium, i.e., the function which satisfies Eq. (3) with $q=\delta\left(x-x_{o}\right) \delta\left(\mu-\mu_{o}\right) \delta\left(t-t_{o}\right)$, is trivially expressible as

$$
\begin{aligned}
G & =\Psi_{i}\left(x, \mu ; t-t_{o}\right) & & t>t_{o} . \\
& =0 & & t<t_{o} .
\end{aligned}
$$

\section{CONCLUSION}

It has been seen that a varied set of neutron transport problems can be treated in a uniform manner with the present method. The approach is the analogy of the classical separation of variables method for partial differential equations. The resulting "functions" are certainly more singular, but not any more difficult to deal with.

It should be emphasized that while the illustrations have all been extremely 
simple problems, it is expected that the structure of the solutions for more complicated situations will be similar. Thus, consider the equation

$$
\frac{\partial \psi}{\partial t}+\mathbf{\nabla} \cdot \nabla \psi+v \sigma \psi=\int F\left(\mathbf{v}, \mathbf{v}^{\prime}\right) \psi\left(\mathbf{r}, \mathbf{v}^{\prime}, t\right) d \mathbf{v}^{\prime} .
$$

Qualitatively we expect the following type of eigenvalue spectrum: First a continuum corresponding to each point $\mathbf{v}$ in velocity space where $F\left(\mathbf{v}, \mathbf{v}^{\prime}\right) \neq 0$. These "eigenfunctions" will all be singular. In addition, there may be a set of discrete eigenvalues corresponding to well-behaved functions. It is expected that the total set of eigenfunctions will be complete in both position and velocity. Moreover, there will be various orthogonality properties.

The simplifications utilized in this paper serve primarily to permit certain integrals to be found explicitly. No particular limitations of principle seem to have been made. In complicated problems where the explicit functions may not be readily evaluated, the present method may serve as a basis of approximation-for example, one could expand in terms of a finite number of the elementary solutions.

\section{APPENDIX A}

It is illuminating to see how the formal solution of the integral equation for completeness given in Section IV reduces in the case $\alpha=-1, \beta=+1$ to that obtained by a trick in Section II.

In Section IV we considered an arbitrary function $\psi(\mu)(-1 \leqq \mu \leqq+1)$. The expansion coefficients $a_{n \pm}$ and $A(\nu)$ are to be determined by

$$
\frac{c}{2} \nu A(\nu)=N^{+}-N^{\top} \text {. }
$$

Here

$$
N(z)=\frac{1}{X(z)} \frac{1}{2 \pi i} \int_{-1}^{1} \frac{c}{2} \frac{\mu^{\prime} \psi^{\prime}\left(\mu^{\prime}\right) X^{-}\left(\mu^{\prime}\right)}{\left[\lambda\left(\mu^{\prime}\right)-(i \pi / 2) c \mu^{\prime}\right]\left(\mu^{\prime}-z\right)}
$$

where

$$
\psi^{\prime}\left(\mu^{\prime}\right)=\psi\left(\mu^{\prime}\right)-a_{0+} \phi_{0+}\left(\mu^{\prime}\right)-a_{0-} \phi_{0-}\left(\mu^{\prime}\right) .
$$

The $a_{0 \pm}$ are determined by the conditions

$$
\int_{-1}^{1} \mu^{l} \frac{\left[\mu \psi^{\prime} X(\mu)\right]}{\lambda(\mu)-(i \pi / 2) c u} d \mu=0, \quad l=0,1 .
$$

The appropriate $X(z)$ is:

$$
X(z)=\frac{e^{\Gamma(z)}}{1-z^{2}}
$$


with

$$
\Gamma(z)=\frac{1}{2 \pi i} \int_{-1}^{1} \frac{\ln G\left(\mu^{\prime}\right) d \mu^{\prime}}{\mu^{\prime}-z}
$$

Now note that

$$
G(\mu)=\frac{1-c \mu \tanh ^{-1} \mu+(i \pi / 2) c \mu}{1-c \mu \tanh ^{-1} \mu-(i \pi / 2) c \mu}=\frac{\left(1-c z \tanh ^{-1} z\right)_{+}}{\left(1-c z \tanh ^{-1} z\right)_{-}} .
$$

Hence

$$
\Gamma(z)=\frac{1}{2 \pi i} \int_{c} \ln \frac{1-c z^{\prime} \tanh ^{-1} z^{\prime}}{z^{\prime}-z} d z^{\prime}
$$

where the contour $c$ encircles a branch cut from -1 to +1 in the clockwise direction.

The form of (A.8) suggests the use of the Cauchy integral formula. Since $1-c z^{\prime} \tanh ^{-1} z^{\prime} \rightarrow 1-c$ as $z^{\prime} \rightarrow \infty$, it is convenient to take $\left(1-c z^{\prime} \tanh ^{-1} z^{\prime}\right) /$ $(1-c)$ as the argument of the logarithm in (A.8). $[\Gamma(z)$ is unchanged since the additions above and below the cut cancel.] The resulting logarithm will not be analytic in the cut plane because of singularities-at the zeros of $1-c z \tanh ^{-1} z$. The function

$$
\ln \left\{\frac{1-c z^{\prime} \tanh ^{-1} z^{\prime}}{\left(\nu_{o}^{2}-z^{2}\right)(1-c)}\right\}
$$

is analytic. However, the argument of the logarithm now does not tend to 1 as $z^{\prime} \rightarrow \infty$. This is remedied by multiplying by $1-z^{2}$. Thus

$$
\Gamma(z)=\frac{1}{2 \pi i} \int_{c} \ln \left[\frac{\left(1-z^{\prime 2}\right.}{\nu_{o}^{2}-z^{\prime 2}} \frac{1-c z^{\prime} \tanh ^{-1} z^{\prime}}{1-c}\right] \frac{d z^{\prime}}{z^{\prime}-z} .
$$

Completing the contour with a large circle at infinity and using the Cauchy integral formula gives

$$
\Gamma(z)=\ln \left[\frac{1-z^{2}}{\nu_{o}^{2}-z^{2}} \frac{1-c z \tanh ^{-1} z}{1-c}\right]
$$

or

$$
X(z)=\frac{1}{\nu_{o}^{2}-z^{2}} \frac{1-c z \tanh ^{-1} z}{1-c}
$$

In particular

$$
X^{-}(\mu)=\frac{1}{\nu_{o}^{2}-\mu^{2}} \frac{\left[1-c u \tanh ^{-1} \mu-(i \pi / 2) c \mu\right]}{1-c} .
$$


and therefore

$$
N(z)=\frac{\nu_{o}^{2}-z^{2}}{1-c z \tanh ^{-1} z} \frac{1}{2 \pi i} \int_{-1}^{1} \frac{\mu \psi^{\prime}(\mu) d \mu}{\left(\nu_{o}^{2}-\mu^{2}\right)(\mu-z)} .
$$

with the conditions of (A.4). To reduce this to the form of Section II, we note that the two conditions are equivalent to

$$
\int_{-1}^{1} \frac{\mu \psi^{\prime}(\mu) d \mu}{\nu_{o} \mp \mu}=\int_{-1}^{1} \mu \phi_{o \pm}(\mu) \psi^{\prime}(\mu) d \mu=0
$$

This form of the conditions together with the partial fraction decomposition

$$
\begin{aligned}
{\left[\frac{1}{\nu_{o}^{2}-\mu^{2}}\right]\left[\frac{1}{\mu-z}\right]=\frac{1}{2 \nu_{0}} } & {\left[\frac{1}{\left(\nu_{o}-z\right)\left(\nu_{o}-\mu\right)}+\frac{1}{\left(\nu_{o}-z\right)(\mu-z)}\right.} \\
& \left.+\frac{1}{\left(\nu_{o}+z\right)(\mu-z)}=\frac{1}{\left(\nu_{o}+z\right)\left(\nu_{o}+\mu\right)}\right]
\end{aligned}
$$

shows that

$$
\int_{-1}^{1} \frac{\mu \psi^{\prime}(\mu) d \mu}{\left(\nu_{o}^{2}-\mu^{2}\right)(\mu-z)}=\frac{1}{\nu_{o}^{2}-z^{2}} \int_{-1}^{1} \frac{\mu \psi^{\prime}(\mu) d \mu}{\mu-z}
$$

Hence

$$
N(z)=\frac{1}{1-c z \tanh ^{-1} z} \frac{1}{2 \pi i} \int_{-1}^{1} \frac{c}{2} \frac{\mu \psi^{\prime}(\mu) d \mu}{\mu-z} .
$$

Equations (A.18) and (A.15) are just the results of Section II.

\section{APPENDIX B}

An essential point in the general completeness proof of Section IV was that it must be possible to choose the coefficients $a_{o \pm}$ so that the necessary conditions for a solution of the integral equation are satisfied. Let us check this for the case $\alpha=0, \beta=+1$. (To be definite it is assumed that $c$ is less than one.)

It must be shown that

$$
M_{o+}=\int_{0}^{1} \frac{\mu X^{-}(\mu)}{\lambda(\mu)-(i \pi / 2) c \mu} \phi_{o+}(\mu) d \mu \neq 0 .
$$

Since $\phi_{o+}(\mu) \sim 1 /\left(\nu_{o}-\mu\right)$, it is enough to show that

$$
R(z)=\int_{0}^{1} \frac{\mu X^{-}(\mu)}{\lambda(\mu)-(i \pi / 2) c \mu} \frac{d \mu}{(z-\mu)}
$$

does not vanish for $z$ real in the range $1<z<\infty$. 
In the present case

$$
X(z)=\frac{e^{\Gamma(z)}}{1-z}
$$

with

$$
\Gamma(z)=\frac{1}{2 \pi i} \int_{0}^{1} \frac{\ln G\left(\mu^{\prime}\right) d \mu^{\prime}}{\mu^{\prime}-z}
$$

Therefore,

$$
\begin{aligned}
\Gamma^{\sim}(\mu) & =\frac{1}{2 \pi i}\left[P \int_{0}^{1} \frac{\ln G\left(\mu^{\prime}\right) d \mu^{\prime}}{\mu^{\prime}-\mu}-\pi i \ln G(\mu)\right] \\
& =\frac{1}{\pi} P \int_{0}^{1} \frac{\theta\left(\mu^{\prime}\right) d \mu^{\prime}}{\mu^{\prime}-\mu}-\frac{1}{2} \ln G(\mu) .
\end{aligned}
$$

Therefore,

$$
\begin{aligned}
X^{-}(\mu) & =\frac{G(\mu)^{1 / 2}}{1-\mu} \exp \frac{P}{\pi} \int_{0}^{1} \frac{\theta\left(\mu^{\prime}\right) d \mu^{\prime}}{\mu^{\prime}-\mu} \\
& =\sqrt{\frac{\lambda-(i \pi / 2) c \mu}{\lambda+(i \pi / 2) c \mu}} \frac{1}{1-\mu} \exp \frac{P}{\pi} \int_{0}^{1} \frac{\theta\left(\mu^{\prime}\right) d \mu^{\prime}}{\mu^{\prime}-\mu}
\end{aligned}
$$

Hence,

$$
R(z)=\int_{0}^{1} \frac{\mu d \mu}{1-\mu} \frac{1}{\sqrt{\lambda^{2}+\left(\pi^{2} c^{2} \mu^{2}\right) / 4}} \frac{\exp \frac{P}{\pi} \int_{0}^{1} \frac{\theta\left(\mu^{\prime}\right) d \mu^{\prime}}{\mu^{\prime}-\mu}}{(z-\mu)} .
$$

We see that for $z$ real and in the range $1<z<\infty, R(z)$ is an integral of a positive definite quantity and thus does not vanish.

RECEIved: May 22, 1959

\section{REFERENCES}

1. N. G. Van Kampen, Physica 21, 949 (1955).

2. N. I. Muskhelishvili, "Singular Integral Equations." Noordhoff, Groningen, 1953.

3. K. M. Case, Annals of Physics 8, 349 (1959). 